\title{
ACCESS CONTROL AND INTERLOCK SYSTEM AT THE ADVANCED PHOTON SOURCE CONF-970503--
}

\author{
J. Forrestal, R. Hogrefe, M. Knott, W. McDowell, D. Reigle, L. Solita, R. Koldenhoven, D. Haid \\ Advanced Photon Source, Argonne National Laboratory, \\ 9700 South Cass Avenue, Argonne, Illinois USA 60439
}

\section{Abstract}

The Advanced Photon Source (APS) consists of a linac, position accumulator ring (PAR), booster synchrotron, storage ring, and up to 70 experimental beamlines. The Access Control and Interlock System (ACIS) utilizes redundant programmable logic controllers (PLCs) and a third hard-wired chain to protect personnel from prompt radiation generated by the linac, PAR, synchrotron, and storage ring. This paper describes the ACIS's design philosophy, configuration, hardware, functionality, validation requirements, and operational experience.

\section{INTRODLCTION}

Personnel protection from prompt radiation produced by the APS accelerators is provided using four PLC-based ACISs. The first system covers the linac/PAR, the second the booster synchrotron, the third the if area of the storage ring (designated Zone $F$ ), and the fourth covers the remaining zones (A-E) of the storage ring. In addition, each of the 70 beamlines uses PLCs for their Personnel Protection Systems (PSSs). The PSSs interface to the Zone F ACIS and storage ring ACIS. This paper describes the four ACISs.

\section{DESIGN PHILOSOPHY}

All the ACISs are designed to the same criteria:

- they must fail-safe so that any single fault leaves the system in a safe beam-inhibiting state,

- they offer redundant and independent protection so that any component or subsystem failure leaves the system in a safe state,

- they monitor beam enclosure tunnel security and prevent beam operations if any unsafe or potentially unsafe condition is detected, and

- they incorporate built-in testing to facilitate biannual validation of all functions.

PLCs are special-purpose microprocessor-based controllers which interface to "real-world" input and output devices. They monitor the input devices, execute userwritten logic, and update output devices based on the result of these logic functions. Allen-Bradley PLC5 series PLCs were chosen to implement the ACIS logic for several reasons:

- higher reliability than comparable relay-based systems,

- ease of programming and self documenting,

- built-in features for testing, system monitoring, and diagnostics.

- easy interfacing of external control devices,
- modular design, wide variety of compatible devices, and

- field proven operation, mature technology.

\section{CONFIGURATION}

Each ACIS monitors its specific accelerator and communicates with its neighboring ACISs. Figure 1 illustrates the relationship among the four ACISs, their relationship to each other, and then the relationship to the PSSs. Two independent PLC chains (A and B) and a third, "hardwired" chain $C$, monitor tunnel security and system integrity. If any system trips, the equipment controlled by the ACISs is disabled and beam is inhibited. Controlled equipment consists of rf amplifiers, modulators, steering/ focusing magnet power supplies, and the electron gun.

The ACISs also send permissives to partitions which are installed between the accelerators. Closed partitions prevent beam from entering a downstream area or prevent radiation from "back streaming" to an upstream area. The partition between the linac/PAR and synchrotron consists of three movable beam blocks. Each block is monitored by both chains of the linac/PAR and synchrotron ACIS. Permits to open are granted by both ACISs as long as each machine is in the same operating mode. The partition between the synchrotron and Zone $F$ consists of steering magnets which direct the beam to a dump or towards Zone $F$ for injection into the ring. The status of these magnets is sensed by magnetic gap switches installed in the magnet and by current monitors installed on the outputs of the magnet's power supplies. This partition is monitored by the Zone F ACIS (both chains).

Ultimately 70 beamlines will connect to the storage ring. The front-ind sction of a beamline is located inside the storage ring's tunnel and contains photon and safety shutters. When closed, these shutters protect the experimental area outside the storage ring wall from stored beam radiation. The closed positions of the shutters are monitored by both PLC chains and their status is sent to the beamlines for use by the PSSs.

\section{HARDWARE}

ACIS hardware consists of emergency shutdown buttons, controlled access key switch $s$, radiation monitors, beam current monitors, limit switches, relays, watchdog timers, control panels, message displays, magnetic locks, and controlled equipment interface panels. The PLC processors are installed in the main control room $1(\mathrm{CR})$ and are connected to remote input/output $(\mathrm{H} / \mathrm{O})$ crates via fiber optic cables. All racks containing ACIS equipment are DISTRAMTNON OF THS DOCUMENT IS UNAMTED

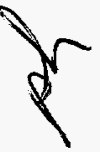

The submitted manuscript has been created by the University of Chicago as Operator of Argonne National Laboratory ("Argonne") under Contract No. W-31-109-ENG-38 with the U.S. Department of Energy. The U.S. Government retains for itself, and others acting on its behalf, a paid-up, nonexclusive. irrevocabie worldwide license in said article to reproduce. prepare derivative works. tiscribute copies to the public, and perform publicly and display publicly, by or on behalf of the Government. 


\section{DISCLAIMER}

This report was prepared as an account of work sponsored by an agency of the United States Government. Neither the United States Government nor any agency thereof, nor any of their employees, make any warranty, express or implied, or assumes any legal liability or responsibility for the accuracy, completeness, or usefulness of any information, apparatus, product, or process disclosed, or represents that its use would not infringe privately owned rights. Reference herein to any specific commercial product, process, or service by trade name, trademark, manufacturer, or otherwise does not necessarily constitute or imply its endorsement, recommendation, or favoring by the United States Government or any agency thereof. The views and opinions of authors expressed herein do not necessarily state or reflect those of the United States Government or any agency thereof. 


\section{DISCLAMMER}

Portions of this document may be illegible in electronic image products. Images are produced from the best available original document. 


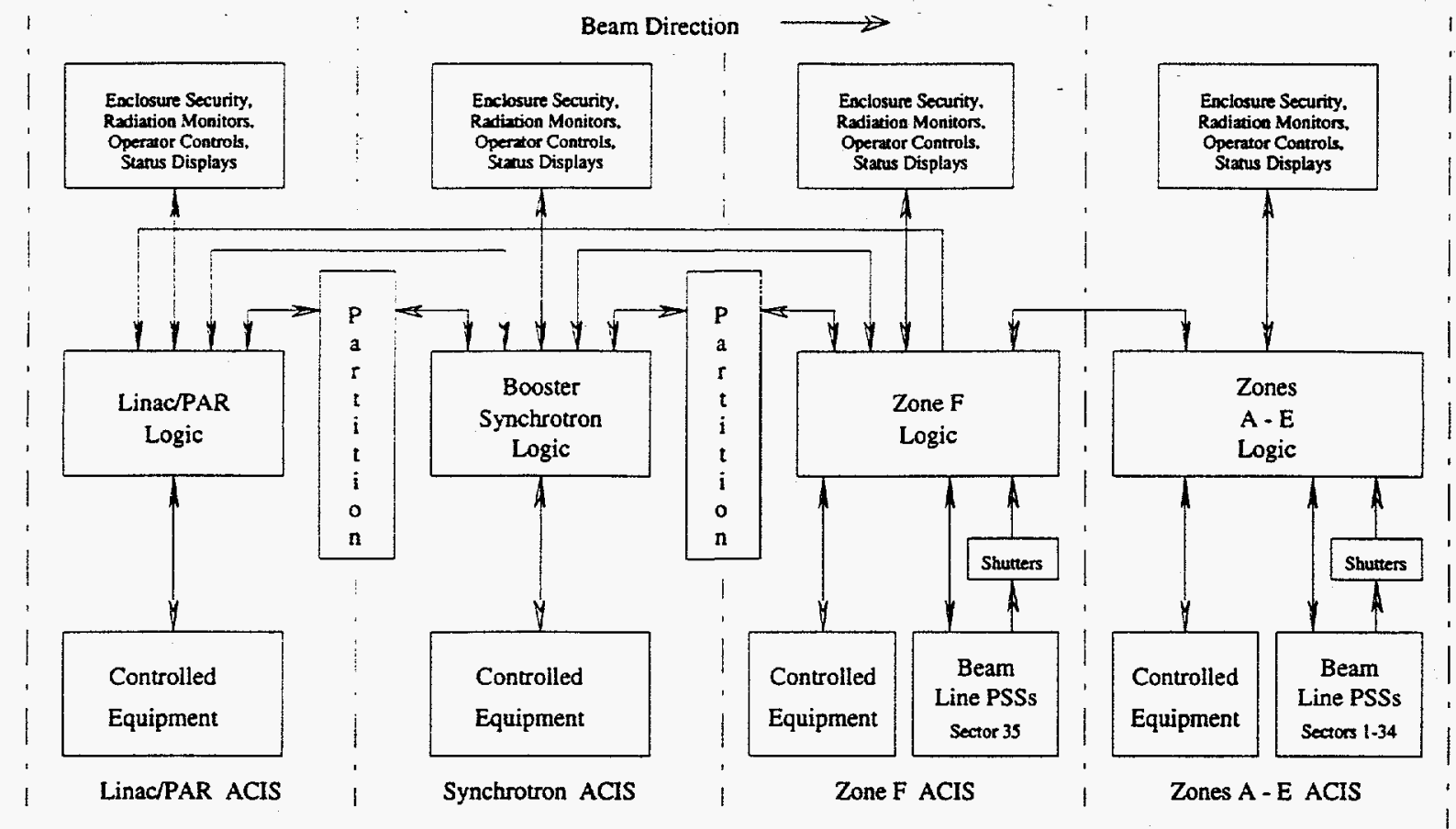

Figure 1: ACIS configuration (one chain only).

locked and tamper-monitored. ACIS cables are labeled and protected by separate cable tray and conduit.

Figure 2 is a typical interface to the controlled equipment. Enables are granted the equipment if two normallyopen relay contacts wired in series are closed. The relay coils are independently driven by chain $A$ and chain $B$. In addition to asserted PLC outputs, other conditions must be met before the relays energize: chain $C$ (the hard-wired chain) must be complete, a beam permit signal from the MCR must be on, and watchdog timers must be energized. For testing and system validation, key-operated switches are installed to direct the ACIS permissives from "real" controlled equipment to test loads.

The MCR beam permit signal is driven by a key switch on the ACIS control panel. It is monitored by the PLC and independently sends a pulse train to the receivers in the interface panel. If the pulses are present, the contacts, which are in series with the output relays, close and the relays are energized. The watchdog timers receive pulses from their respective PLC chain via a local output module. The contacts are closed as long as the PLC programs are running. If a program ceases to execute its periodic scan, the pulses stop, the contacts open, and the output relays de-energize.

\section{FUNCTIONALITY}

As shown in Figure 3, the ACISs are state machines with the five operating modes. The modes escalate from Authorized Access to Beam Permit, and have the following meaning:

AUTHORIZED ACCESS MODE: tunnel access is allowed on an unrestricted basis. It is entered upon initial program execution, a system reset, a normal transition from Controlled Access Mode, or a trip.
SEARCH / VERIFICATION MODE: A search is enforced to verify no personnel are in the beam enclosure tunnels. As the tunnel is searched, buttons located on the beam shutdown station (BSS) boxes are pressed to indicate that area was visited by the search team. The search must be completed in a preset time. If the time is exceeded, or if a button is hit out of order, a trip to Authorized Access Mode is generated.

CONTROLLED ACCESS MODE: This mode is entered after the successful completion of the tunnel search. It is implemented to allow personnel into the tunnels on a limited basis without requiring another search. While in the tunnels, personnel are required to carry a controlled access key. If any key is out, the ACIS cannot transit to the next higher mode. This mode may be re-entered from Beam Permit Pending Mode or by a Controlled Access Mode trip.

Beam Permit Pending Mode: Entry from Controlled Access Mode requires a 1-minute warning message initiated by the MCR operator. This message is announced in the tunnel by audible alerts and red strobe beacons installed in the BSS boxes. At the end of the warning, the alerts silence but the strobes remain flashing.

BEAM PERMIT MODE: To enter this mode the MCR operator inserts a key in the beam permit key switch and selects the "Enable" position. The PLCs monitor this switch and, if all other conditions are met, energize the ACIS output relays. At the same time, a pulse train is transmitted to the beam permit receivers in the output relay panels.

Authorized Access or Controlled Access Modes are unconditionally entered if a fault is detected. The types of faults are listed in Figure 3. 


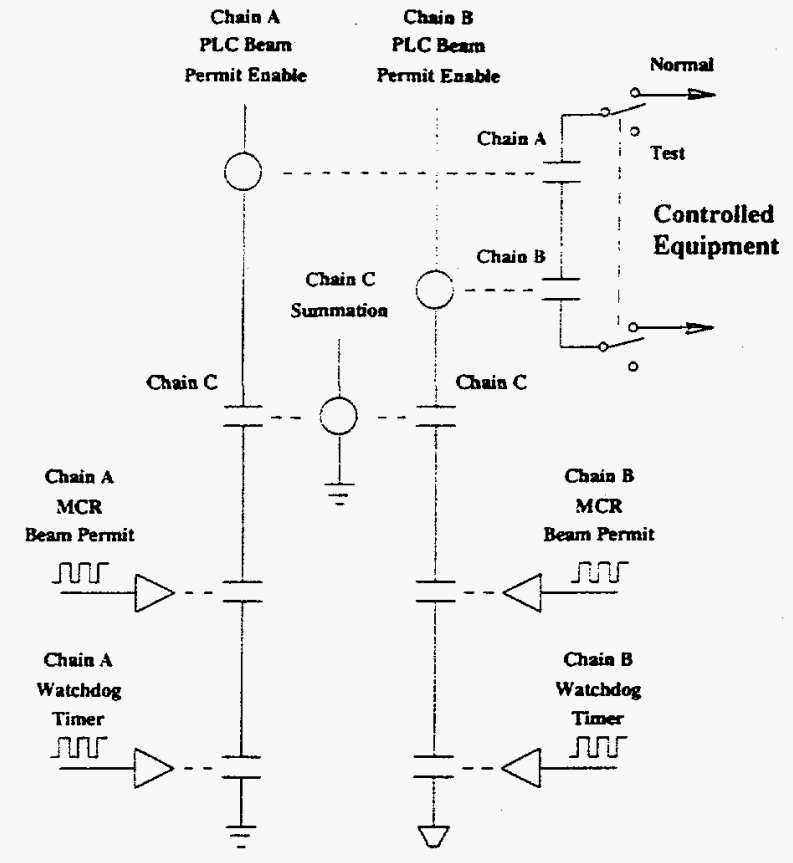

Figure 2: ACIS output interface.

Permits to open the partitions and permissives to energize the controlled equipment are only granted by the ACISs. Other commands to open the partitions or energize equipment must be sent by MCR operators. For additional protection, the ACIS monitors the state of the controlled equipment. If the feedback signal indicates the equipment is on while being disabled, or if the interface cable is disconnected, the ACIS declares a "No Shutdown Verification" alarm. This alarm locks access doors to prevent entry and activates the in-tunnel strobes to alert personnel to evacuate.

The ACISs are designed to eliminate the use of physical jumpers for testing. A test mode-which can only be entered if all sets of controlled equipment are disconnected and a test mode key-operated switch is on-is provided to allow bypassing of certain functions. Since chains $A$ and $B$ cross trip each other and chain $C$ influences the final per-. missives, the validation procedure must prove system independence. Test features include disabling influences from other chains, providing a "quick search," inhibiting watchdog timers, and masking tampers.

\section{VALIDATION REQUIREMENTS}

The ACISs are validated every six months or whenever the interlock software is modified. The PLC processors calculate checksums unique to their application programs. These checksums are used to identify the program version, and any change to the checksum requires revalidation of the system. ACIS validation involves exercising all $\mathrm{L} / \mathrm{O}$ devices and verifying that the interlock logic performs as specified, ACIS status is properly displayed, and the controlled equipment shuts down when disabled. Validation results are recorded in the validation test proce- dure along with the checksum of the software being validated. Completed procedures are archived in the APS's Document Control Center.

\section{OPERATIONAL EXPERIENCE}

The linac/PAR ACIS became operational in July 1993, the synchrotron ACIS (including most Zone F hardware) in February 1994, and the Zone F and storage ring ACISs in February 1995. To date there have been no failures of PLC hardware. However, some external devices have failed, namely an intermittent relay socket, a failed watchdog timer, sluggish operation of a beam stopper's control cylinder, and intermittent trips from a radiation monitor. Every ACIS challenge has resulted in a successful shutdown, and no unsafe failures have occurred.

\section{CONCLUSION}

The four ACISs were designed and installed over a four-year period based on the APS's construction schedule. PLC system modularity and flexibility was vital in meeting construction and commissioning goals and provided full protective functionality during commissioning. The troubleshooting capabilities of the PLCs are valuable tools in diagnosing system operation and correcting malfunctions. The PLC-based ACISs have exceeded all operational expectations.

\section{ACKNOWLEDGMENTS}

Work is supported by the U.S. Department of Energy, Office of Basic Energy Sciences, under Contract No. W31-109-ENG-38.

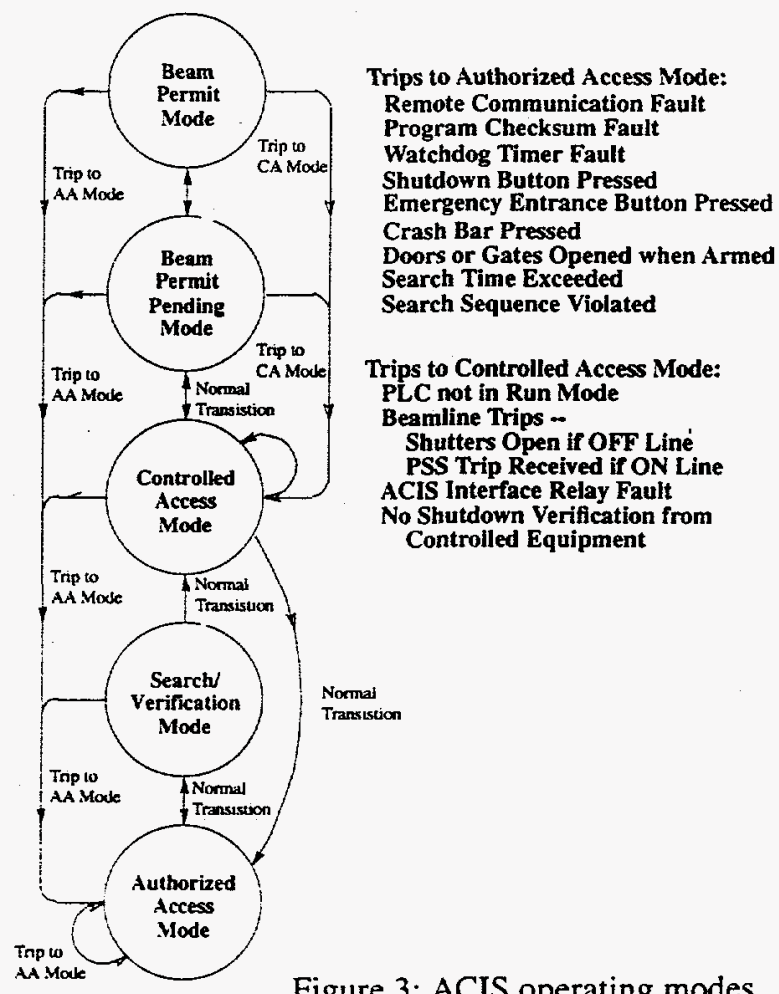

Figure 3: ACIS operating modes. 